\title{
Light Dispersion in Diamond-like Crystals
}

\author{
Vladimir Rumyantsev, 1 \\ ${ }^{1}$ Department of Theory of Complex Systems Dynamic Properties, A. A. Galkin Institute for Physics \& Engineering, Donetsk, Ukraine \\ ${ }^{2}$ Mediterranean Institute of Fundamental Physics, Rome, Italy
}

Email address:

vladimir.rumyantsev2011@yandex.ru

\section{To cite this article:}

Vladimir Rumyantsev. Light Dispersion in Diamond-like Crystals. Journal of Photonic Materials and Technology.

Vol. 5, No. 1, 2019, pp. 16-23. doi: 10.11648/j.jmpt.20190501.14

Received: May 2, 2019; Accepted: June 3, 2019; Published: June 18, 2019

\begin{abstract}
Dispersion of light in diamond-like crystals is investigated. Dispersion laws of exciton polaritons in this structures, which (apart from the diamond itself) include silicon and germanium is obtained within the quasi-molecular model of valent crystals. Dispersion curves point to the fact that in the vicinity of exciton resonance under small damping one must account for the exciton-photon interaction. The calculation shows that in a certain frequency range the existence of an additional light wave is possible. The dispersion laws of exciton polaritons in a diamond-like structure in the vicinity of frequency of the lowest dipole transition of a crystalline quasi-molecule (a $\sigma$-bond) are obtained.
\end{abstract}

Keywords: Diamond-like Crystals, Light Dispersion, Exciton Polaritons

\section{Introduction}

A crystal is a many-electron system, which in some cases can be treated as an assembly of several strongly localized subsystems. The attempt to account for correlation between electrons as accurately as possible underlies the group function method. The efficiency of this method (provided that the conditions for strong orthogonality have been properly taken into account) depends essentially on the successful initial choice of electronic groups for the subsequent description of the full electronic system of a crystal. Valent crystals, in particular, can be considered from a standpoint of the concept of localized two-electron bonds. In such a case the main interest lies in group functions dependent on two electrons (geminals), since the electronelectron correlation is primarily the result of a pairwise interaction. The study of electron correlation in atoms and molecules permits to conclude that the most essential effects are caused by interaction between electrons with opposite spins, whose orbitals are localized at the same region (i.e., for instance, closed atomic $S$-shells as well as $\sigma$-bonds in molecules). The use of two-electron functions for the description of molecular $\sigma$-bonds was first proposed by $\mathrm{V}$. A. Fock [1].

K. B. Tolpygo has developed a quasi-molecular model of a diamond-like valent crystal, which permitted to give a consistent interpretation of the ground and excited states in crystals based on the analysis of $\sigma$-bonds between neighboring atoms as well as to construct the crystal lattice dynamics [2]. Within the quasi-molecular model a strong bond in a diamond-like crystal is formed by the overlapping of wave functions of electrons with opposite spins (hence the term "valent crystals"). When atoms consolidate into a crystal the orbitals of valent electrons of each atom (stretched towards its four nearest neighbors) are formed by $s p^{3}$ hybridization of atomic functions (see Figures 1,2).

Diamond-like crystals, which (apart from the diamond itself) include silicon and germanium, are featured by a complex elementary cell (Figure 1), containing eight atoms. The crystal lattice of these semiconductors can be viewed as a superposition of two face-centered cubic lattices shifted with respect to each other along a cube's diagonal through the 1/4 -th of its length. Each atom of such a crystal is bound to its four neighbors, each bond being directed along a tetrahedron's edge (i.e. along the $\langle 111\rangle$-direction of the cubic lattice).

Experimental studies show that (see e.g. [3]) the bulk of valent charge of such crystals is concentrated in the vicinity of centers of tetrahedral $\sigma$-bonds. When proceeding from a quasi-molecule (a $\sigma$-bond) to examination of a crystal one finds that the relative change of lengths, elastic constants and binding energies amounts to approximately $1 \%$. In addition, measurements show that the Compton profiles of $C-C$ 
bonds differ insignificantly from the profiles of such bonds in a $\mathrm{C}_{2} \mathrm{H}_{6}$ gas [4]. Therefore, experimental data indicate in fact that the properties of a valent (unexcited) bond are practically independent of its environment (with the exception for the nearest neighbors) similarly to the quasi-independence of one-electron states in the Hartree-Fock model

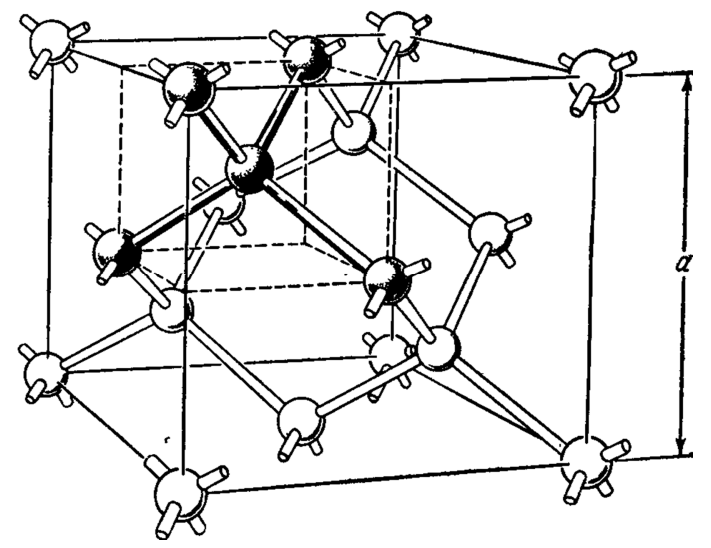

Figure 1. The scheme of the elementary cell of a diamod-like crystal ( ${ }^{a}$ is the lattice constant).

The charge-density distribution of valent electrons calculated within the quasi-molecular model agrees closely with experimental results $[2,5]$. The estimates for the binding energy of the crystal lattice, the compressibility, and the linear thermal expansion coefficient also favor the quasimolecular model [6]. Within this model a diamond-like crystal is viewed as an aggregation of weakly interacting structural units (SU) - $\sigma$-bonds. Based on the above, in this study we use a model according to which a crystal with a diamond-like structure is a set of weakly interacting structural units (SU) - $\sigma$-bonds. Thus, when calculating the local electromagnetic field in such a crystal, it becomes possible to use the technique developed in for molecular crystals [7-9].

\section{Theoretical Background}

A diamond-like crystal constituted by quasimolecules (bonds) has an fcc-lattice with the basis formed by four quasi-molecules (see Figure 1). Each molecule is exposed to the self-consistent field of all other SU's of the crystal.

We shall assume an SU's interaction to be a dipole-dipole one (with the exception for the nearest surrounding of each $\mathrm{SU})$ so that it can be described by the internal field tensor $\hat{\varphi}^{s s^{\prime}}$ obtained within the dipole approximation. Interaction of nearest SU's strongly differs from that of point dipoles and requires a more accurate description. Hence the amplitude of the electric field strength $\mathbf{E}_{s}^{\omega, \mathbf{k}}$ created at the point $\mathbf{r}_{s}^{l}$ by all SU's of the crystal (except for the nearest surrounding) has the form:

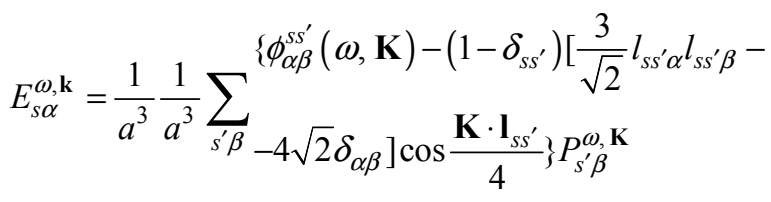

The second term in Eq. (1) is the field of the nearest neighbors of the $\mathbf{r}_{s}^{l}$-th SU written in dipole approximation.

The explicit form of the Fourier-transform of tensor $\hat{\varphi}^{s s^{\prime}}$ has been obtained (with the account for interaction delay) through Ewald transformation in Refs. [9] [10]. Within the long-wave approximation $\hat{\phi}^{\text {ss' }}(\omega, \mathbf{k})$ can be represented as an expansion in wave vector $\mathbf{K}$ (with an accuracy up to the second order):

$$
\begin{aligned}
& \phi_{\alpha \beta}^{s s^{\prime}}\left(K_{0}, \mathrm{~K}\right)=-2 \pi \frac{K_{\alpha} K_{\beta}-K_{0} \delta_{\alpha \beta}}{K^{2}-K_{0}^{2}}+\frac{2 \pi}{3} \delta_{\alpha \beta}-\left(1-\delta_{s s^{\prime}}\right)\left(4.33385 \delta_{\alpha \beta}+0.18238 l_{s s^{\prime} \alpha}^{2} \delta_{\alpha \beta}-1.80757 l_{s s^{\prime} \alpha} l_{s s^{\prime} \beta}\right) \\
& -\delta_{s s^{\prime}}\left(0.23708 K^{2} \delta_{\alpha \beta}-0.28998 K_{\alpha} K_{\beta}-0.42126 K_{\alpha}^{2} \delta_{\alpha \beta}\right)-\left(1-\delta_{s s^{\prime}}\right)\left\{0.08696\left(l_{s s^{\prime} \alpha} l_{s s^{\prime} \beta}-\frac{8}{3} \delta_{\alpha \beta}\right)\left(\mathrm{K} \cdot 1_{s s^{\prime}}\right)^{2}\right. \\
& -0.09904\left[\mathrm{~K} \cdot 1_{s s^{\prime}}\left(l_{s s^{\prime} \alpha} K_{\beta}+K_{\alpha} l_{s s^{\prime} \beta}\right)-\frac{2}{3} \delta_{\alpha \beta}\left(\mathrm{K} \cdot 1_{s s^{\prime}}\right)^{2}\right]+0.04717 K^{2}\left[\left(l_{s \alpha}+l_{s^{\prime} \beta}\right)\left(l_{s \beta}+l_{s^{\prime} \alpha}\right)+2\left(l_{s \alpha} l_{s^{\prime} \beta}+l_{s \beta} l_{s^{\prime} \alpha}\right)\right] \\
& -0.02903\left[\mathrm{~K} \cdot\left(1_{s}+1_{s^{\prime}}\right)\right]^{2}\left[\begin{array}{l}
3\left(l_{s \alpha}+l_{s^{\prime} \alpha}\right)\left(l_{s \beta}+l_{s^{\prime} \beta}\right)- \\
-2\left(l_{s \alpha} l_{s^{\prime} \beta}+l_{s^{\prime} \alpha} l_{s \beta}\right)-\frac{16}{3} \delta_{\alpha \beta}
\end{array}\right]-0.05540\left(1_{s}+l_{s^{\prime}}\right) \cdot[\mathrm{KK}]^{+}\left[\begin{array}{l}
\left(l_{s \alpha}+l_{s^{\prime} \alpha}\right)\left(l_{s \beta}+l_{s^{\prime} \beta}\right)- \\
-2\left(l_{s \alpha} l_{s^{\prime} \beta}+l_{s^{\prime} \alpha} l_{s \beta}\right)-\frac{8}{3} \delta_{\alpha \beta}
\end{array}\right] \\
& +\frac{2}{3} \delta_{\alpha \beta}\left[0.14167\left(1_{s}+1_{s^{\prime}}\right) \cdot[\mathrm{KK}]^{+}+0.03761\left(\mathrm{~K} \cdot\left(1_{s}+1_{s^{\prime}}\right)\right)^{2}\right]-\left(l_{s \alpha}+l_{s^{\prime} \alpha}\right)\left[0.14167[\mathrm{KK}]_{\beta}^{+}+0,03761 \mathrm{~K} \cdot\left(1_{s}+1_{s^{\prime}}\right) K_{\beta}\right] \\
& \left.-\left(l_{s \beta}+l_{s^{\prime} \beta}\right)\left[0.14167[\mathrm{KK}]_{\alpha}^{+}+0.03761 \mathrm{~K} \cdot\left(1_{s}+1_{s^{\prime}}\right) K_{\alpha}\right]+\sum_{\gamma}\left|e_{\alpha \beta \gamma}\right|\left[0.05611[\mathrm{KK}]_{\gamma}+0,17161 \mathrm{~K} \cdot\left(1_{s}+1_{s^{\prime}}\right) K_{\gamma}\right]\right\}
\end{aligned}
$$

where $[\mathrm{KK}]_{\alpha}^{+} \equiv \sum_{\beta \gamma}\left|e_{\alpha \beta \gamma}\right| K_{\beta} K_{\gamma}, e_{\alpha \beta \gamma}$ is the anti-symmetric third-rank unit pseudo-tensor, vectors $\mathbf{l}_{s}(\mathrm{~s}=1 \ldots 4)$ define the directions of bonds $\mathbf{l}_{1}=(1,1,1), \mathbf{l}_{2}=(1,-1,-1)$,
$\mathbf{I}_{3}=(-1,1,-1), \mathbf{l}_{4}=(-1,-1,1), \mathbf{l}_{s s^{\prime}}=\mathbf{l}_{s}-\mathbf{l}_{s^{\prime}}$ (see Figure 2).

Let us analyze in more detail the propagation of electromagnetic excitation in diamond-like valent crystals in the energy region of formation of the deep Frenkel exciton. 
The use of the quasi-molecular model permits to apply to diamond-like structures the technique developed previously for molecular crystals [8,9]. According to the model [2], each pair of electrons forming a $\sigma$-bond between neighboring atoms, together with the quarters of atoms' skeletons, which make up a part of the bond, are viewed as a quasi-molecule. It should be highlighted that the manyelectron approach, employed here, based on Hartree approximation for geminals, and taking into account their time dependence, is different from the chaotic phases approximation used in [11] in that the former allows for the exchange and correlation within each bond. It should be noted here that in molecular crystals the subject of investigation were the "true" excited states (whose lifetime is limited only by radiation), whereas in diamond-like crystals excitons are metastable [12] - the excitation levels fall within the continuous spectrum $\hbar \omega>E_{G}$ ( $E_{G}$ is the band gap width).

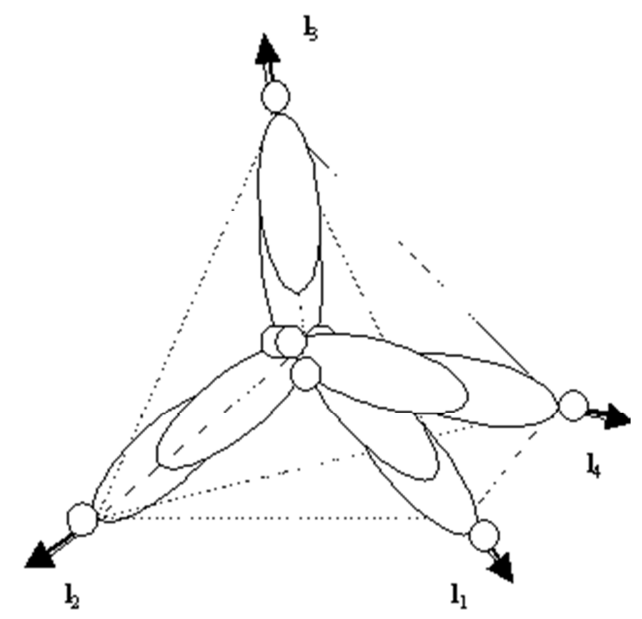

Figure 2. Coordination tetrahedron of a diamond-like crystal.

The specifics of interaction between electromagnetic waves and a solid medium is defined by the latter's response function. Our primary task consists therefore in finding this function for the case of diamond-like crystals.

Within the quasi-molecular model of valent crystals each pair of electrons forming a $\sigma$-bond between neighboring atoms is viewed as a "molecule", whose state $\Psi_{\sigma}^{l}\left(\overrightarrow{\mathbf{r}}_{1}, \mathbf{r}_{2}\right)$ is described by a two-electron Schredinger equation:

$$
\frac{\partial \Psi_{\sigma}^{l}}{\partial t}=\left(\hat{\mathrm{H}}_{\sigma}^{l}+\hat{W}_{\sigma}^{l}\right) \Psi_{\sigma}^{l}
$$

where $l$ is the cell number, $\sigma$ is the quasi-molecule number in the cell, $H_{S}^{l}$ is the Hamiltonian, which includes static Coulomb field of the rest of SU's being at the ground state $\Psi_{\sigma 0}^{l}$. Interaction between all other SU's ( $\sigma$-bonds) can be considered as a dipole-dipole one with the exception for the nearest neighbors of each SU. In the latter case it is different from the interaction between point dipoles and hence is subject to refinement. There is a perturbation exerted upon the $\mathbf{r}_{\sigma}^{l}$-th bond by all other bonds, which arises due to the partial virtual excitation of SU's and can be represented in the following form:

$$
\hat{W}_{\sigma}^{l}=-\mathrm{P}_{\sigma}^{l} \cdot \mathrm{E}\left(t, \mathrm{r}_{\sigma}^{l}\right)+\Delta \hat{W}_{\sigma}^{l}
$$

where $\mathbf{P}_{\sigma}^{l}=\mathbf{r}_{1}+\mathbf{r}_{2}$ is the dipole moment operator, $\mathbf{E}\left(t, \mathbf{r}_{\sigma}^{l}\right)$ is the field exerted upon the $\left(\begin{array}{l}l \\ \sigma\end{array}\right)$-th bond by all other bonds with the exception for the nearest surrounding, whose contribution is taken into account by the term $\Delta \hat{W}_{\sigma}^{l}$. Interaction of a $\sigma$-bond with the nearest surrounding cannot be treated as a dipole-dipole one since the distance between centers of neighboring bonds is smaller than the bond length itself.

According to Ref. [2] one can adopt a concept according to which every SU has its eigen-functions $\Psi_{\sigma i}^{l}$, which satisfy a Hartree-type equation $\hat{H}_{\sigma}^{l} \Psi_{\sigma i}^{l}=E_{\sigma i} \Psi_{\sigma i}^{l}$ (where $E_{\sigma i}$ are the energy eigen-values of a quasi-molecule). Such an approach is valid for moderate excitation levels as long as wave functions $\Psi_{\sigma i}^{l}$ can be considered as localized at a single bond. If the non-stationary state of the bond is written in the form (1. 1. 23) in Ref [9] then it is easy to show that coefficients $c_{\sigma i}^{l}$ corresponding to excitations with index $i$ satisfy (approximately) the following equations:

$$
\begin{gathered}
\dot{c}_{\sigma i}^{l}(t)=i \mathbf{P}_{i 0}^{\sigma} \cdot \mathbf{E}\left(\mathbf{r}_{\sigma}^{l}, t\right) \exp \left(i \omega_{i 0} t\right)- \\
-i \sum_{\sigma^{\prime} l^{\prime}}^{\prime} \sum_{j}\left\{\begin{array}{l}
c_{\sigma^{\prime} j}^{l^{\prime}}(t) \exp \left[i\left(\omega_{i 0}-\omega_{j 0}\right) t\right]+ \\
+\left(c^{*}\right)_{\sigma^{\prime} j}^{l}(t) \exp \left[i\left(\omega_{i 0}+\omega_{j 0}\right) t\right]
\end{array}\right\} I_{i j}^{\sigma \sigma^{\prime}} \\
\left(\dot{c}^{*}\right)_{\sigma i}^{l}(t)=-i \mathbf{P}_{0 i}^{\sigma} \cdot \mathbf{E}^{*}\left(\mathbf{r}_{\sigma}^{l}, t\right) \exp \left(-i \omega_{i 0} t\right)+ \\
+i \sum_{\sigma^{\prime} l^{\prime}}^{\prime} \sum_{j}\left\{\begin{array}{l}
\left(c^{*}\right)_{\sigma^{\prime} j}^{l^{\prime}}(t) \exp \left[-i\left(\omega_{i 0}-\omega_{j 0}\right) t\right]+ \\
+c_{\sigma^{\prime} j}^{l^{\prime}}(t) \exp \left[-i\left(\omega_{i 0}+\omega_{j 0}\right) t\right]
\end{array}\right\}\left(I^{*}\right)_{i j}^{\sigma \sigma^{\prime}}
\end{gathered}
$$

where the dipole moment operator matrix is $\mathbf{P}_{i 0}^{\sigma} \equiv \int\left(\Psi^{*}\right)_{\sigma i}^{l}(1,2) \hat{\mathbf{P}} \Psi_{\sigma 0}^{l}(1,2) d \mathbf{r}_{1} d \mathbf{r}_{2} \quad, \quad \omega_{i 0} \equiv E_{\sigma i}-E_{\sigma 0}$, (from here on we use the atomic system of units), and:

$$
I_{i j}^{\sigma \sigma^{\prime}} \equiv 4 \int \frac{\Psi_{\sigma i}^{l}(1,2) \Psi_{\sigma 0}^{l}(1,2) \Psi_{\sigma^{\prime} 0}^{l^{\prime}}(3,4) \Psi_{\sigma^{\prime} j}^{l^{\prime}}(3,4)}{\left|\mathbf{r}_{1}-\mathbf{r}_{3}\right|} \prod_{m=1}^{4} d \mathbf{r}_{m}
$$

Wave functions $\Psi_{\sigma i}^{l}$ are chosen to be real-valued, whereas summation in (5) and (6) is carried out over the nearest neighbors of the $\mathbf{r}_{\sigma}^{l}$-th SU. Since the Fourier-amplitude $\mathbf{P}_{\sigma}^{\omega, \mathbf{k}}$ of the average dipole moment SU equals to:

$$
\mathbf{P}_{\sigma}^{\omega, \mathbf{k}}=\sum_{i} \mathbf{P}_{0 i}^{\sigma}\left[c_{\sigma}^{\mathbf{k}}\left(\omega+\omega_{i 0}\right)+c_{\sigma}^{-\mathbf{k}}\left(\omega_{i 0}-\omega\right)\right] \equiv \sum_{i} \mathbf{P}_{0 i}^{\sigma} C_{i}^{\sigma, \mathbf{k}}(\omega)
$$


by performing the Fourier transformation of the system (5)(6) and keeping in mind that we consider the region in the vicinity of the dipole transition $(i=f)$ we arrive at the system of equations in $C_{i}^{\sigma, \mathbf{k}}(\omega)$ (the real parts of the Fourier-transforms of coefficients $\left.c_{\sigma i}^{l}(t)\right)$ :

$$
\begin{aligned}
& C_{i \neq f}^{\sigma, \mathrm{k}}(\omega)=\frac{2 \omega_{i 0}}{\omega_{i 0}^{2}-\omega_{f 0}^{2}}\left\{\mathrm{P}_{o i}^{\sigma} \cdot \mathrm{E}_{\sigma}^{\omega, \mathrm{k}}-\sum_{\sigma^{\prime}}^{\prime}\left[I_{i f}^{\sigma \sigma^{\prime}} C_{f}^{\sigma^{\prime}, \mathrm{k}}(\omega)+\sum_{j \neq f} I_{i j}^{\sigma \sigma^{\prime}} C_{j}^{\sigma^{\prime}, \mathrm{k}}(\omega)\right] 2 \cos \frac{\mathrm{k} \cdot 1_{\sigma \sigma^{\prime}}}{4}\right\} \\
& C_{f}^{\sigma, \mathbf{k}}(\omega)=\frac{2 \omega_{f o}}{\omega_{f o}^{2}-\omega^{2}}\left\{\mathbf{P}_{o f}^{\sigma} \cdot \mathbf{E}_{\sigma}^{\omega, \mathbf{k}}-\sum_{\sigma^{\prime}}^{\prime}\left[I_{f f} C_{f}^{\sigma^{\prime}, \mathbf{k}}(\omega)+\sum_{j \neq f} I_{f j}^{\sigma \sigma^{\prime}} C_{j}^{\sigma^{\prime}, \mathbf{k}}(\omega)\right] 2 \cos \frac{\mathbf{k} \cdot \mathbf{l}_{\sigma \sigma^{\prime}}}{4}\right\}
\end{aligned}
$$

Here $\mathbf{k}$ is the dimensionless wave vector, $\mathbf{l}_{\sigma \sigma^{\prime}}=\mathbf{l}_{\sigma}-\mathbf{l}_{\sigma^{\prime}}$, $\mathbf{l}_{1}=(1,1,1), \mathbf{l}_{2}=(1,-1,-1), \mathbf{l}_{3}=(-1,1,-1), \mathbf{l}_{4}=(-1,-1,1)$ are vectors directed along the bonds (Figures 1. 3, 1. 4). Since coefficients $C_{i}^{\sigma, \mathbf{k}}$ in the right-hand side of Eq. (9) are small as compared to unity the system of equations (9), (10) can be solved by the iteration method, where in the zeroth approximation:

$$
C_{i \neq f}^{\sigma, \mathbf{k}}=\frac{2 \omega_{i o}}{\omega_{i o}^{2}-\omega_{f o}^{2}} \mathbf{P}_{i o}^{\sigma} \cdot \mathbf{E}_{\sigma}^{\omega, \mathbf{k}}
$$

It should be noted however that the coefficient with $C_{f}^{\sigma^{\prime}, \mathbf{k}}$ in the right-hand side of Eq. (10) is not small due to a resonant denominator and hence upon substitution of (11) into (10) the latter is solved exactly with respect to $C_{f}^{\sigma, \mathbf{k}}$. Symbolically Eq. (10) can be written as $\hat{X} \cdot\left(\hat{I}+\hat{X}^{-1} \cdot \hat{\gamma}\right) \hat{C}_{f}=\hat{Y} \quad$. When inverting the matrix $\hat{X} \cdot\left(\hat{I}+\hat{X}^{-1} \cdot \hat{\gamma}\right)$ one should remember that it is factorable in the actual domain of $\mathbf{k}$ and hence

$$
\hat{C}_{f}=\left(\hat{I}-\hat{X}^{-1} \cdot \hat{\gamma}\right) \cdot \hat{X}^{-1} \cdot \hat{Y}
$$

The corresponding matrix elements in equality (12) have the form:

$$
\begin{gathered}
\gamma_{\sigma \sigma^{\prime}}=\frac{4 I_{f f}}{\omega_{f o}}\left(1-\frac{4 I_{f f}}{\omega_{f o}}-\Omega^{2}\right)^{-1} \sin \frac{\mathbf{k} \cdot \mathbf{l}_{\sigma}}{4} \sin \frac{\mathbf{k} \cdot \mathbf{l}_{\sigma^{\prime}}}{4} \\
Y^{\sigma}=\frac{2}{\omega_{f o}}\left(1-\frac{4 I_{f f}}{\omega_{f o}}-\Omega^{2}\right)^{-1} \times\left\{\mathbf{P}_{o f}^{\sigma} \cdot \mathbf{E}_{\sigma}^{\omega, \mathbf{k}}-\sum_{\sigma^{\prime}}^{\prime} \mathbf{E}_{\sigma^{\prime}}^{\omega, \mathbf{k}} \cdot \cos \frac{\mathbf{k} \cdot \mathbf{l}_{\sigma \sigma^{\prime}}}{4} \sum_{j \neq f} \frac{4 I_{f j}^{\sigma \sigma^{\prime}}}{\omega_{j o}} \mathbf{P}_{o j}^{\sigma^{\prime}}\left(1-\frac{\omega_{f o}^{2}}{\omega_{j o}^{2}}\right)^{-1}\right. \\
\left(\hat{X}^{-1}\right)_{\sigma \sigma^{\prime}}=\delta_{\sigma \sigma^{\prime}}-\frac{4 I_{f f}}{\omega_{f o}}\left[1+\frac{4 I_{f f}}{\omega_{f o}}\left(1+f_{c}\right)-\Omega^{2}\right]^{-1} \cos \frac{\mathbf{k} \cdot \mathbf{l}_{\sigma}}{4} \cos \frac{\mathbf{k} \cdot \mathbf{l}_{\sigma^{\prime}}}{4}
\end{gathered}
$$

where $f_{c}=\cos \frac{k_{x}}{2} \cos \frac{k_{y}}{2} \cos \frac{k_{z}}{2}$, and as above $\Omega \equiv \omega / \omega_{f o}$ and $\hat{I}$ being the unity matrix. Substituting the solution of system of equations (9), (10) into (8), we get:

$$
P_{\sigma, \alpha}^{\omega, \mathbf{k}}=\sum_{\sigma^{\prime}, \beta} a^{3} A_{\alpha \beta}^{\sigma \sigma^{\prime}}(\omega, \mathbf{k}) E_{\sigma^{\prime}, \beta}^{\omega, \mathbf{k}}
$$

Tensor $\hat{A}^{\sigma \sigma^{\prime}}$ is the dynamic polarizability of the $\left(\begin{array}{l}l \\ \sigma\end{array}\right)$-th bond, whose explicit form (in view of its cumbersomeness) is given in the Appendix (A-2) in Ref. [9]. Dependency of coefficients $A_{\alpha \beta}^{\sigma \sigma^{\prime}}(\omega, \mathbf{k})$ on $\mathbf{k}$ is explained by the fact that our analysis does not entirely fits within the frames of the dipoledipole approximation. Taking into complete account an interaction of the $\left(\begin{array}{l}l \\ \sigma\end{array}\right)$-th SU with its nearest neighbors results in a situation when for the symmetry $D_{3 d}$ of a diamond-like crystal the dipole $A_{1 g} \rightarrow A_{2 u}, A_{1 g} \rightarrow E_{u}$ cease to be forbidden for certain higher odd multipolar moments (the latter is the cause for the dependency of $A_{\alpha \beta}^{\sigma \sigma^{\prime}}(\omega, \mathbf{k})$ on the wave vector $\mathbf{k}$ ).

The Fourier-amplitude of the field acting upon the $\left(\begin{array}{l}l \\ \sigma\end{array}\right)$-th SU of the crystal is found by the procedure described in Ref. [13],

$$
E_{\sigma, \alpha}^{\omega, \mathbf{k}}=a^{-3} \sum_{\sigma^{\prime}, \beta} \tilde{\phi}_{\alpha \beta}^{\sigma \sigma^{\prime}}(\omega, \mathbf{k}) P_{\sigma^{\prime}, \beta}^{\omega, \mathbf{k}}
$$

Here tensor $\hat{\tilde{\phi}}^{\sigma \sigma^{\prime}}$ serves to denote the expression in angular brackets in Eq. (1. 2. 16) [9]. The solvability condition for the system of linear homogeneous equations (16) and (17), with the account for (1. 2. 17) [9], yields the dispersion law $\omega=\omega(\mathbf{k})$, which characterizes exciton polaritons in crystals of the considered type.

In the general case (16), (17) constitute a system of 24 
equations, which is factorized under $\mathbf{k} \rightarrow 0$ and upon both sides of equalities being subjected to a unitary transformation. Analysis of equations yielded by the transformation shows that in the limit $\mathbf{k} \rightarrow 0$ only the $\Gamma_{2}^{\prime}, \Gamma_{15}$-symmetry excitations are set in motion (which interact with the electromagnetic wave). The so found limiting exciton frequencies are determined (through coefficients $Z_{i j}$, see Appendix A-2 [9]) by the whole state spectrum of geminals. Finding of the latter constitutes a separate problem; here we shall confine ourselves to a simpler model.

\section{Results and Discussion}

Let us consider an approximation where each valent bond of the crystal in addition to the ground state $\Psi_{\sigma, o}^{l}$ has only one excited state described by a wave function $\Psi_{\sigma, f}^{l}$ of the same form as in the study [14], which is constructed on the basis of the same $s p^{3}$-hybrids as the ground state in the study [6]. It is easy to verify that in this case the Fourier-transform (10) $C_{f}^{\sigma, \mathbf{k}}(\omega)$ has the form:

$$
C_{f}^{\sigma, \mathrm{k}}(\omega)=\frac{2}{\omega_{f o}\left(\Omega_{1}^{2}-\Omega^{2}\right)}\left\{\left[1-\left(1-\Omega_{1}^{2}\right) f_{\sigma \sigma}(\Omega, \mathrm{K})\right] \mathrm{P}_{o f}^{\sigma} \cdot \mathrm{E}_{\sigma}^{\omega, \mathrm{k}}-\left(1-\Omega_{1}^{2}\right) \sum_{\sigma^{\prime}}^{\prime} f_{\sigma \sigma^{\prime}}(\Omega, \mathrm{K}) \mathrm{P}_{o f}^{\sigma^{\prime}} \cdot \mathrm{E}_{\sigma^{\prime}}^{\omega, \mathrm{k}}\right\}
$$

where:

$$
\begin{gathered}
f_{\sigma \sigma^{\prime}}=\left(\Omega_{1}^{2}-\Omega^{2}\right)^{-1} \sin \frac{\mathrm{K} \cdot 1_{\sigma}}{4} \sin \frac{\mathrm{K} \cdot 1_{\sigma^{\prime}}}{4}+\left(\Omega_{2}^{2}-\Omega^{2}\right)^{-1} \cos \frac{\mathrm{K} \cdot 1_{\sigma}}{4} \cos \frac{\mathrm{K} \cdot 1_{\sigma^{\prime}}}{4} \\
\Omega_{1}^{2}=1-4 I_{f f} / \omega_{f o} \\
\Omega_{2}^{2}=1+4\left[1+2 \cos \left(K_{1} / 2\right) \cos \left(K_{2} / 2\right) \cos \left(K_{3} / 2\right)\right] I_{f f} / \omega_{f o}
\end{gathered}
$$

Therefore the dynamic polarizability $A_{\alpha \beta}^{\sigma \sigma^{\prime}}(\omega, \mathbf{k})$ assumes the form:

$$
A_{\alpha \beta}^{\sigma \sigma^{\prime}}(\omega, \mathbf{k})=\frac{\Delta_{\|}}{3} \frac{l_{\sigma \alpha} l_{\sigma^{\prime} \beta}}{\Omega_{1}^{2}-\Omega^{2}}\left\{\delta_{\sigma \sigma^{\prime}}\left[1-\left(1-\Omega_{1}^{2}\right) f_{\sigma \sigma}(\Omega, \mathbf{K})\right]-\left(1-\delta_{\sigma \sigma^{\prime}}\right)\left(1-\Omega_{1}^{2}\right) f_{\sigma \sigma^{\prime}}(\Omega, \mathbf{K})\right\} \equiv \frac{1}{3} a_{\sigma \sigma^{\prime}}(\omega, \mathbf{k}) l_{\sigma \alpha} l_{\sigma^{\prime} \beta}
$$

Here $\Delta_{\|}$is $\Delta_{\|}=2 P_{f o}^{\sigma} P_{o f}^{\sigma} / \omega_{f o}$. The fact that the longitudinal polarization of the bond is much bigger than the transversal one can be accounted for by introducing variables $P_{\sigma}, E_{\sigma}$ :

$$
P_{\sigma \alpha}^{\omega, \mathbf{k}}=P_{\sigma} l_{\sigma \alpha}, E_{\sigma}=\frac{1}{3} \sum_{\alpha} E_{\sigma \alpha}^{\omega, \mathbf{k}} l_{\sigma \alpha}
$$

This procedure permits to substantially reduce the number of variables and hence the number of equations in system (16), (17). In terms of the new variables $P_{\sigma}, E_{\sigma}$ this system of equations assumes the form:

$$
\begin{aligned}
& \left\{\begin{array}{l}
P_{\sigma}=\sum_{\sigma^{\prime}} a^{3} a_{\sigma \sigma^{\prime}}(\omega, \mathbf{k}) E_{\sigma^{\prime}} \\
E_{\sigma}=\sum_{\sigma^{\prime}} a^{-3} \Phi_{\sigma \sigma^{\prime}}(\omega, \mathbf{k}) P_{\sigma^{\prime}}
\end{array}\right. \\
& P_{\Gamma_{2}^{\prime}}=\frac{\Delta_{\|} a^{3}}{\Omega_{2}^{2}-\Omega^{2}}\left(E_{\Gamma_{2}^{\prime}}+\frac{1-\Omega_{1}^{2}}{\Omega_{1}^{2}-\Omega^{2}} \frac{1}{8}[\mathbf{K K}]^{+} \cdot \mathbf{E}_{\Gamma_{15}}\right), \\
& E_{\Gamma_{2}^{\prime}}=a^{-3}\left\{\left[a_{0}-\left(a_{1}+\frac{a_{3}-a_{4}}{3}\right) K^{2}\right] P_{\Gamma_{2}^{\prime}}-\frac{a_{3}}{3}[\mathbf{K K}]^{+} \cdot \mathbf{P}_{\Gamma_{15}}\right\} \\
& \mathbf{P}_{\Gamma_{15}}=\frac{\Delta_{\|} a^{3}}{\Omega_{1}^{2}-\Omega^{2}}\left\{\mathbf{E}_{\Gamma_{15}}+\frac{1-\Omega_{1}^{2}}{\Omega_{2}^{2}-\Omega^{2}} \frac{1}{8}[\mathbf{K K}]^{+} \cdot E_{\Gamma_{2}^{\prime}}-\frac{1-\Omega_{1}^{2}}{\Omega_{1}^{2}-\Omega^{2}} \frac{1}{4} \mathbf{K}\left(\mathbf{K} \cdot \mathbf{E}_{\Gamma_{15}}\right)\right\} \\
& \mathbf{E}_{\Gamma_{15}}=\frac{1}{3} a^{-3}\left\{\left(\frac{8 \pi}{3}-a_{0}+a_{1} K^{2}\right) \mathbf{P}_{\Gamma_{15}}-8 \pi \frac{\mathbf{K}\left(\mathbf{K} \cdot \mathbf{P}_{\Gamma_{15}}\right)-\mathbf{P}_{\Gamma_{15}} \Omega^{2} / M}{K^{2}-\Omega^{2} / M}-a_{2} P_{\Gamma_{2}^{\prime}}[\mathbf{K K}]^{+}+a_{3} \mathbf{K}\left(\mathbf{K} \cdot \mathbf{P}_{\Gamma_{15}}\right)-a_{4} \mathbf{P}_{\Gamma_{15}} ; \mathbf{K K}\right\}
\end{aligned}
$$


Here $\Gamma_{2}^{\prime}, \Gamma_{15}$ are the indices of irreducible representations of the wave vector group, coefficients $a_{i}(i=0 \ldots 4)$, which reflect the structure of a diamond-like crystal are given in Table $1, M=c^{2} / a^{2} \omega_{f 0}^{2}$. Vector $\mathbf{P}_{\Gamma_{15}}$ has the meaning of the dipole moment of a cell $\mathbf{P}=\left(P_{1}, P_{2}, P_{3}\right)$, where $\mathbf{P}=2 \mathbf{P}_{\Gamma_{15}}$ (by analogy with $\mathbf{E}_{\Gamma_{15}}$ ). Higher electronic excitations are taken into account by introduction of the dielectric permeability $\varepsilon_{\infty}$. In this case the resonant term of the dimensionless polarizability of a quasimolecule-bond should be supplemented by a high-frequency term $A_{\infty}$ :

$$
A_{\infty}=3\left(\varepsilon_{\infty}-1\right)\left[8 \pi\left(\varepsilon_{\infty}+2-\delta\right)\right]^{-1}
$$

where $\delta=3 a_{0}\left(\varepsilon_{\infty}-1\right) / 8 \pi$ is the structural correction due to the local field. The existence condition of a non-trivial solution of the system (24) yielded first of all [15] the limiting $(\mathbf{k} \rightarrow 0)$ excitonic frequencies with the account for the higher excitations:

$$
\begin{aligned}
& \Omega_{\Gamma_{2}^{\prime}}^{2}=\Omega_{2}^{2}-a_{0} \Delta_{\|}, \\
& \Omega_{\|}^{2}(0)=\Omega_{1}^{2}+\frac{\Delta_{\|}}{3}\left(\frac{16 \pi}{3}+a_{0}\right)\left[1+A_{\infty}\left(\frac{16 \pi}{3}+a_{0}\right)\right]^{-1} \\
& \Omega_{\perp}^{2}(0)=\Omega_{1}^{2}-\frac{\Delta_{\|}}{3}\left(\frac{8 \pi}{3}-a_{0}\right)\left[1-A_{\infty}\left(\frac{8 \pi}{3}-a_{0}\right)\right]^{-1}
\end{aligned}
$$

Numerical values of the squares of limiting frequencies for diamond and silicon crystals obtained within the accurate description of interaction between nearest $\sigma$-bonds as well as the constants necessary for their evaluation are given in Table 2. For comparison purposes Table 3 gives the same frequencies calculated within the approximation where only the dipole-dipole interaction is present between a crystal's SU's (including their immediate surrounding). Tables 2 and 3 show the importance of as exact as possible account for interaction between nearest neighbors in each crystalline SU.

Table 1. Structural coefficients $a_{i}$.

\begin{tabular}{lllll}
\hline $\boldsymbol{a}_{0}$ & $\boldsymbol{A}_{\boldsymbol{1}}$ & $\boldsymbol{a}_{2}$ & $\boldsymbol{A}_{3}$ & $\boldsymbol{A}_{4}$ \\
\hline 5,15599 & 0,35397 & 0,54053 & 2,11659 & 0,12445 \\
\hline
\end{tabular}

Table 2. Squares of limiting frequencies.

\begin{tabular}{lllllll}
\hline Crystal & $\boldsymbol{\varepsilon}_{\infty}$ & $\boldsymbol{\Delta}_{\|}$ & $\boldsymbol{I}_{f f} / \boldsymbol{\omega}_{f o}$ & $\boldsymbol{\Omega}_{\Gamma_{2}^{\prime}}^{2}$ & $\boldsymbol{\Omega}_{\|}^{2}$ & $\boldsymbol{\Omega}_{\perp}^{2}$ \\
\hline Diamond & 5,7 & 0,08 & 0,067 & 1,40 & 0,89 & 0,59 \\
Silicon & 11,7 & 0,10 & 0,073 & 1,38 & 0,86 & 0,45 \\
\hline
\end{tabular}

Table 3. Squares of limiting frequencies (dipole-dipole approximation).

\begin{tabular}{llll}
\hline Crystal & $\boldsymbol{\Omega}_{\boldsymbol{\Gamma}_{2}^{\prime}}^{2}$ & $\boldsymbol{\Omega}_{\|}^{2}$ & $\boldsymbol{\Omega}_{\perp}^{2}$ \\
\hline Diamond & 2,82 & 0,83 & 0,17 \\
Silicon & 3,27 & 0,79 & $-0,03$ \\
\hline
\end{tabular}

Table 4. Values of limiting exciton frequencies.

\begin{tabular}{lllllllll}
\hline & $\boldsymbol{\Omega}_{\mid \boldsymbol{|}}(\mathbf{0})$ & $\boldsymbol{\Omega}_{\perp}(\mathbf{0})$ & $\boldsymbol{\omega}_{\boldsymbol{f} \mathbf{0}}(\mathbf{a} . \mathrm{e})$ & $\boldsymbol{\omega}_{\boldsymbol{f} \mathbf{0}}(\mathrm{eV})$ & $\boldsymbol{\omega}_{\boldsymbol{f} \mathbf{0}}\left(\mathbf{c}^{-1}\right)$ & $\boldsymbol{a}(\boldsymbol{M})$ & $\boldsymbol{M}$ \\
\hline Diamond & 0,94 & 0,77 & 0,97 & 26,38 & $4,02 \cdot 10^{16}$ & $1,78 \cdot 10^{-10}$ & $1,8 \cdot 10^{3}$ \\
Silicon & 0,93 & 0,67 & 0,49 & 13,33 & $2,03 \cdot 10^{16}$ & $2,71 \cdot 10^{-10}$ & $3,0 \cdot 10^{3}$ \\
\hline
\end{tabular}

Detailed calculations employing the technique of Ref. [13] crystals performed in [15] within the model of two-level SU's of diamond-like crystals have led to the following dispersion laws in the vicinity of the dipole transition frequency $\omega_{f o}$ of a quasimolecule. For longitudinal excitons:

$$
\Omega_{\|}^{2}(\mathbf{K})=\Omega_{\|}^{2}(0)+\mathbf{K}^{2}\left[\left(I_{f f} / \omega_{f 0}\right)-\beta_{\|}(\mathbf{s})\left(\Delta_{\|} / 3\right)\left(1+A_{\infty}\left(\frac{16 \pi}{3}+a_{0}\right)\right)^{-1}\right]
$$

For transversal excitons:

$$
\begin{aligned}
& \Omega_{\perp 1}^{2}(\mathbf{K})=\Omega_{\perp}^{2}(0)-\mathbf{K}^{2} \beta_{\perp 1}(\mathbf{s})\left(\Delta_{\|} / 3\right)\left[1-A_{\infty}\left(\frac{8 \pi}{3}-a_{0}\right)\right]^{-1} \\
& \Omega_{\perp 2}^{2}(\mathbf{K})=\Omega_{\perp}^{2}(0)-\mathbf{K}^{2} \beta_{\perp 2}(\mathbf{s})\left(\Delta_{\|} / 3\right)\left[1-A_{\infty}\left(\frac{8 \pi}{3}-a_{0}\right)\right]^{-1}
\end{aligned}
$$

where $\beta_{\|}(\mathbf{s})=a_{1}+a_{3}+a_{4}\left(s_{1}^{4}+s_{2}^{4}+s_{3}^{4}\right), \quad \beta_{\perp 1}=a_{1}, \beta_{\perp 2}(\mathbf{s})=a_{1}-a_{4}\left(1-s_{1}^{4}-s_{2}^{4}-s_{3}^{4}\right)$.

From the solvability condition of Eqs. (16) and (17) follows the dispersion laws of exciton polaritons $\Omega_{p}=\Omega_{p}(\mathbf{k})$ in a diamond-like structure in the vicinity of frequency $\omega_{f 0}$ of the lowest dipole transition of a crystalline quasi-molecule 
$\left(\Omega=\omega / \omega_{f 0}\right.$ in dimensionless units):

$$
\Omega_{p( \pm)}^{2}=\frac{1}{2}\left\{\Omega_{\|}^{2}(\mathbf{k})+\frac{M \mathbf{k}^{2}}{\varepsilon_{\infty}} \pm \sqrt{\left[\Omega_{\|}^{2}(\mathbf{k})+\frac{M k^{2}}{\varepsilon_{\infty}}\right]^{2}-4 M k^{2} \frac{\Omega_{\perp}^{2}(\mathbf{k})}{\varepsilon_{\infty}}}\right\}
$$

Dispersion laws $\Omega_{\|}(\mathbf{k}), \Omega_{\perp(1,2)}(\mathbf{k})$ of longitudinal and transversal excitons are given by formulas (27) and (28), $M=c^{2} / a^{2} \omega_{f 0}^{2}, a$ is the lattice constant, the values of limiting $(k \rightarrow 0)$ exciton frequencies $\Omega_{\|}(0), \Omega_{\perp}(0)$ and other useful constants are given in Table 2 and Table 4.

Figure 3 shows the graph of $\Omega_{2}-\Omega_{1}$ - the splitting of the excited state 1 of a quasimolecule caused by its interaction only with the nearest SU's ( $\sigma$-bonds). According to formula (19), $\Omega_{2}$ and $\Omega_{1}$ are, correspondingly, the singlet and triplet levels of a quasimolecule. $\Omega_{\Gamma_{2}^{\prime}}$ and $\Omega_{\Gamma_{15}}$ are, correspondingly, the singlet and triplet levels of the crystal [15].

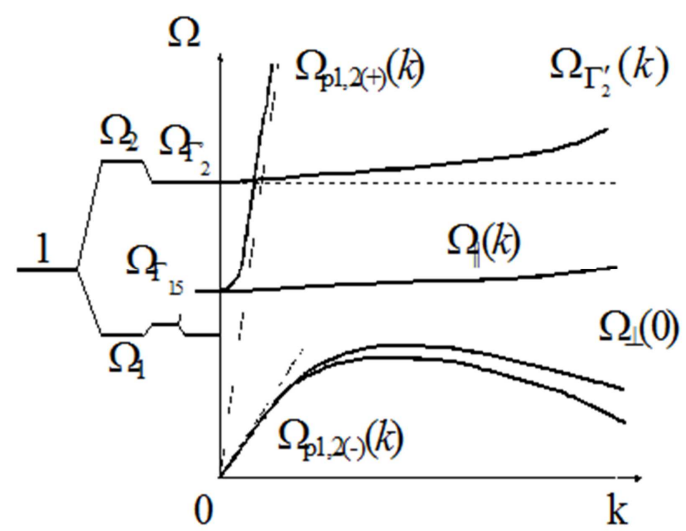

Figure 3. Dispersion curves of electromagnetic excitations in a diamond-like crystal.

\section{Conclusion}

The dispersion curves obtained in the work point to the fact that in the vicinity of exciton resonance under small damping one must account for the exciton-photon interaction and that the $\Gamma_{15}$-symmetry exciton is split into two doubly-degenerated (under $\mathbf{k} \rightarrow 0$ ) branches $\Omega_{p(+)}, \Omega_{p(-)}$, corresponding to a transversal wave and one nondegenerate branch $\Omega_{\|}$, corresponding to a longitudinal wave; in the $\Omega<\Omega_{\perp}$ frequency interval there may exist an additional lightwave.

The electromagnetic wave propagation in symmetric directions $\Delta, \Lambda, \Sigma$ of a diamond-like crystal is investigated. The obtained dispersion relations for exciton polaritons indicate that in the neighborhood of exciton resonance $\Omega_{\perp}(0)$, when $\omega_{\|}-\omega_{\perp}>\Gamma$ ( $\Gamma$ is the damping constant $)$, one must account for the exciton-photon interaction. Whereas the singlet excitonic symmetry level $\Gamma_{2}^{\prime}$ does not "mix" with light, an interaction of the triplet excitonic symmetry level
$\Gamma_{15}$ with light leads to its splitting into one branch corresponding to a longitudinal wave $\omega(\mathbf{k})$ (which does not "mix" with light as well) and two polariton branches $\omega_{-}(\mathbf{k}), \omega_{+1,2}(\mathbf{k})$, whose shape indicates the existence of an additional light wave for $\Omega<\Omega_{\perp}(0)$ in the considered crystals.

Utilization of novel materials for designing of sources of coherent irradiation has become an extensive interdisciplinary scientific area, which includes laser physics, condensed matter physics, nanotechnology, chemistry, and information science [16]. Special attention is paid to possibilities of controlling of propagation of electromagnetic excitations in resulting composite structures such as porous Si (see for example Ref. [17]). The presented results make it possible to expand the possibilities of creating a new class of porous functional materials - polaritonic crystal systems (arrays of microporesresonators) with controlling the propagation of electromagnetic excitations in such composite structures $[18,19]$.

\section{References}

[1] V. A. Fock, "Application of twoelectron functions in the theory of chemical bond", Dokl. Akad. Nauk SSSR, v. 73, No. 4, pp. 735-739, 1950

[2] K. B. Tolpygo. "Two-center wave functions in the theory of valence crystals", Physics of the Solid State, v. 17, No. 6, pp. 1769-1779, 1975.

[3] Y. W. Yang, P. Copens, "On the experimental electron distribution in silicon”, Sol. St. Com., v. 15, No. 9, pp. 15551559, 1974.

[4] W. A. Reed, P. Eisenberger, "Gamma-ray Compton profiles of diamond, silicon and germanium", Phys. Rev. B, v. 6, pp. 4596-4604, 1972.

[5] I. M. Reznik, "Valence electron density in crystals of group IV elements", Physics of the Solid State, v. 19, No. 7. pp. 1983-1986, 1977.

[6] I. M. Reznik, "Adiabatic potential of diamond-like semiconductors in quasi-molecular model", Physics of the Solid State, v. 19, No. 2, pp. 463-468, 1977.

[7] K. B. Tolpygo. "Propagation of light in a crystal as a delayed transmission of excitation of its atoms", Ukrainian Journal of Physics, v. 31, No. 2, pp. 178-187, 1986.

[8] V. V. Rumyntsev, "Dispersion of exciton polaritons in atomic cryocrystals", Ukrainian Journal of Physics, v. 35, No. 12, pp. 1783-1791, 1990.

[9] V. V. Rumyntsev, "Interaction of Electromagnetic Radiation and Light Particles with Imperfect Crystalline Media", NordPress: Donetsk, 2006. 347p. [in Russian]. 
[10] P. P. Ewald, "Die Berechnung optischer und elektrostatischer Getterpotentiale", Ann. Phys. Bd. 64, No. 4, S. 253-287, 1921.

[11] J. A. Van Vechten, R. M. Martin, "Calculation of local effective field: optical spectrum of diamond", Phys. Rev. Lett., v. 28, No. 7, pp. 446-449, 1972.

[12] W. Hanke, L. J. Sham, "Local field and excitonic effects in the optical spectrum of a covalent crystal", Phys. Rev. No. 10, pp. 4501-4511, 1975.

[13] V. V. Rumyntsev, "Optical anisotropy of atomic cryocrystals in the vicinity of exciton resonance", Crystallography Reports, v. 36, No. 6, pp. 1346-1351, 1991.

[14] K. B. Tolpygo, V. M. Shatalov, "The no-current excitation states in homopolar semiconductors", Ukrainian Journal of Physics, v. 20, No. 9, pp. 1476-1483, 1975.

[15] V. V. Rumyntsev, "Optical anisotropy and additional light waves in diamond structure crystals", Ukrainian Journal of Physics, v. 34, No. 9, pp. 1316-1321, 1989.
[16] P. Tighineanu, A. S. Sørensen, S. Stobbe and P. Lodahl, "The Mesoscopic Nature of Quantum Dots in Photon Emission"/ P. Michler (Ed.), "Quantum Dots for Quantum Information Technologies. Nano-Optics and Nanophotonics", Cham: Springer, pp. 165-198, 2017.

[17] Yuri Pivovarenko, "Laser-Induced Fluorescence of Wet Porous Silicon as Laser-Induced Fluorescence of H3O+", Journal of Photonic Materials and Technology, v. 5 (1), pp. 11$15,2019$.

[18] V. V. Rumyntsev, S. A. Fedorov, K. V. Gumennyk, D. A. Gurov, A. V. Kavokin, "Effects of elastic strain and structural defects on slow light modes in a one-dimensional array of microcavities", Superlattices and Microstructures, v. 120, pp. 642-649, 2018.

[19] V. V. Rumyantsev, "Dispersion of electromagnetic excitations in a non-ideal lattice of coupled microcavities containing quantum dots", J. Laser Opt, Photonics, v. 5, p. 38, 2018. 